\title{
Qualidade da intubação endotraqueal em gatos com tiopental sódico, propofol ou tiopental sódico/propofol
}

\section{The quality of endotracheal intubation in cats with thiopentone, propofol or thiopentone/propofol}

\author{
Renata Navarro Cassu, ${ }^{*}$ Lidia Mitsuko Matsubara, ${ }^{* \star}$ Helaine Stevanin, ${ }^{* \star *}$ Gláucia Prada Rodrigues de Barros ${ }^{\star \star \star *}$
}

\section{Resumo}

A obstrução das vias aéreas, produzida pelo aumento das secreções e/ou laringoespasmo, durante a intubação endotraqueal, pode ser um achado freqüente em procedimentos anestésicos realizados em gatos. $O$ objetivo desse estudo foi avaliar a qualidade da intubação endotraqueal (IE) em gatos com o uso de diferentes fármacos na indução anestésica, a fim de estabelecer um protocolo anestésico que possibilite facilitar a mesma. Foram utilizados 10 gatos adultos, com peso médio de $3 \mathrm{~kg}$, clinicamente saudáveis, de ambos os sexos. Esses animais foram avaliados em três protocolos anestésicos diferentes, sendo realizada para todos, medicação préanestésica com acepromazina $0,1 \mathrm{mg} / \mathrm{kg}$ im, 15 minutos antes da indução anestésica, realizada com tiopental sódico a $1,25 \%$ na dose de $12,5 \mathrm{mg} / \mathrm{kg}$ iv (G1), propofol a $1 \%$ na dose de $7 \mathrm{mg} / \mathrm{kg}$ iv (G2) e tiopental sódico a 1,25\% associado ao propofol a $0,7 \%$ na dose de $0,5 \mathrm{ml} / \mathrm{kg}$ de cada agente anestésico iv (G3). Após a indução anestésica foi realizada a IE. Parâmetros avaliados: freqüência e ritmo cardiaco, oximetria de pulso, freqüência respiratória, número de tentativas para a realização da IE, presença de reflexo de tosse, ocorrência de laringoespasmo, coloração das mucosas, grau de relaxamento da glote (escore de $0-2$ ), onde $0=$ relaxamento ruim, $1=$ relaxamento razoável, $2=$ bom relaxamento, necessidade de doses complementares para a IE. A estatística foi realizada com análise de variância de Friedman, teste $Q$ de Cochran's e análise de variância de Tukey. Os resultados obtidos demonstraram melhor relaxamento de glote, maior atenuação do reflexo laringotraqueal, menor incidência de tosse, com menor número de tentativas para a IE, com o uso do propofol (G2), evidenciando a superioridade desse fármaco para a realização de $\mathrm{IE}$ em gatos.

Palavras-chave: gatos, intubação endotraqueal, propofol, tiopental sódico.

\section{Abstract}

Airway obstruction produced by incresead secretions and/or laryngoespasm during intubation is a common finding in cats undergoing anesthesia procedure. This study aimed to investigate the response to endotracheal intubation following thiopentone $(1,25 \%) 12,5 \mathrm{mg} / \mathrm{kg}$ iv (G1), propofol (1\%) $7 \mathrm{mg} / \mathrm{kg}$ iv (G2) or a mixture of thiopentone (1,25\%) and propofol $(0,7 \%) 0,5 \mathrm{ml} / \mathrm{kg}$ iv (G3). The purpose of the study was to ascertain wich of these three induction agents provided the better conditions for endotracheal intubation. Ten adult mongrel cats, weighing 2,5 to $4.0 \mathrm{~kg}$ were premedicated with $0,1 \mathrm{mg} / \mathrm{kg}$ of acepromazine $1 \mathrm{M}$, followed 30 minutes later by induction of anaesthesia. The presence of coughing, apnea, laryngoespasm and glotic relaxation was noted and graded. The number of attempts at intubation and the necessary use of additional indution agent were also recorded. Heart rate, pulse oximetry, respiratory rate were recorded too. Propofol was associated with better results (G2), compared with thiopentone (G1) or propofol/thiopentone (G3). We conclude that, the single use of propofol was superior to thiopentone or thiopentone/propofol as an induction agent for tracheal intubation in the cat.

Keywords: cats, endotracheal intubation, propofol, thiopentone

\section{Introdução}

O aparelho respiratório dos gatos é constituído de estruturas pequenas e delicadas sujeitas a traumas em situações em que o uso da sonda endotraqueal se faz necessário (Taylor,
1994). Além do tamanho reduzido da laringe e da traquéia, os gatos apresentam reflexos protetores bem evidentes, podendo desencadear o laringoespasmo durante o processo de intubação endotraqueal. O laringoespasmo pode persistir por 10 a 20 segundos após o estímulo sobre a laringe (Feldman

\footnotetext{
* Profa. Dra. do Departamento de Anestesiologia Veterinária - Universidade do Oeste Paulista - Faculdade de Ciências Agrárias. Rodovia Raposo Tavares, Km 572. Bairro Limoeiro - Presidente Prudente, SP, CEP 19001-970. email: renavarro@uol.com.br

** Residente do Departamento de Anestesiologia Veterinária - Universidade do Oeste Paulista - Faculdade de Ciências Agrárias. Rodovia Raposo Tavares, Km 572. Bairro Limoeiro - Presidente Prudente, SP, CEP 19001-970.

*** Acadêmica do $5^{\circ}$ ano de Medicina Veterinária - Universidade do Oeste Paulista - Faculdade de Ciências Agrárias. Rodovia Raposo Tavares, Km 572. Bairro Limoeiro - Presidente Prudente, SP, CEP 19001-970.

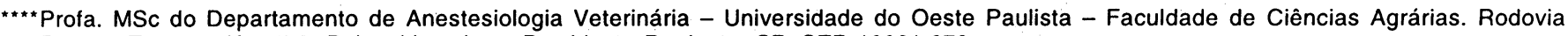
Raposo Tavares, Km 572. Bairro Limoeiro - Presidente Prudente, SP, CEP 19001-970.
} 
et al., 1989). Portanto, para essa espécie, a intubação endotraqueal requer alguns cuidados especiais, a fim de evitar-se maiores problemas decorrentes da mesma (Rex, 1994).

Em algumas circunstâncias, a sonda endotraqueal se faz de extrema necessidade, como em casos de cirurgias em cabeça e pescoço, cirurgias em que a ventilação mecânica seja requerida e em situações em que a regurgitação possa ocorrer, como em cirurgias gastrointestinais (Taylor, 1994).

O trauma advindo das dificuldades encontradas no processo de intubação endotraqueal foi considerado de grande importância, devido a um número significativo de mortalidade em gatos decorrente de obstrução da via respiratória (Clarke e Hall, 1990).

Algumas técnicas podem ser utilizadas, a fim de permitir a intubação endotraqueal na espécie felina, visando minimizar os traumas dela provenientes. A técnica do uso de anestésicos locais é a mais empregada, sendo normalmente feita com spray de lidocaína, desenvolvido para uso humano. $O$ anestésico local pode provocar irritação química da laringe (Watson, 1992; Taylor, 1992, 1993), assim como aumentar a secreção da mucosa traqueal em gatos (Somerville et al., 1990), podendo eventualmente produzir arritmias cardíacas (Heavner, 1986).

O uso do bloqueador neuromuscular, como por exemplo, o suxametônio, tem como objetivo induzir o completo relaxamento da laringe, de modo a permitir uma suave introdução da sonda endotraqueal, porém quando esse método é usado, torna-se essencial a instituição da ventilação controlada (Taylor, 1994).

O uso de plano anestésico profundo não é recomendado, pois para ocorrer o relaxamento da laringe corre-se o risco de uma sobredosagem anestésica (Taylor, 1994).

Tendo em vista melhorar a qualidade de intubação endotraqueal na espécie felina, de modo a reduzir as possíveis seqüelas geradas pelo traumatismo imposto por ela, esse trabalho teve como objetivo comparar a qualidade da intubação endotraqueal em gatos, com o uso de tiopental sódico em relação ao uso do propofol ou tiopental sódico/propofol.

\section{Materiais e métodos}

Após aprovação do Comitê de Ética da Universidade do Oeste Paulista, foram utilizados dez gatos adultos, provenientes do gatil da mesma instituição, com peso médio de $3 \mathrm{~kg} \pm 0,8$, machos e fêmeas, saudáveis, sendo avaliados por meio de exame clínico e exames laboratoriais (hemograma, bioquímico renal e hepático), os quais foram mantidos isolados, durante 30 dias, antes do início do experimento, sendo realizada vermifugação e vacinação dos mesmos.

Esses animais foram avaliados em três protocolos anestésicos diferentes. Na medicação pré-anestésica foi administra$\mathrm{da}$, para todos os grupos, acepromazina ${ }^{\mathrm{a}} 0,1 \mathrm{mg} / \mathrm{kg} \mathrm{im,} 15$ minutos antes da indução anestésica, realizada com tiopental

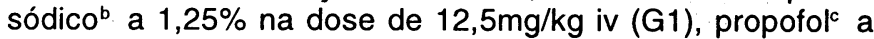
$1 \%$ na dose de $7 \mathrm{mg} / \mathrm{kg}$ iv (G2) e tiopental sódico a $1,25 \%$ associado ao propofol a $0,7 \%$ na dose de $0,5 \mathrm{ml} / \mathrm{kg}$ de cada agente anestésico iv (G3). As doses utilizadas no G3, no qual foi realizada a associação de tiopental/propofol, foram reduzidas em $50 \%$ da dose inicial utilizada no G1 e G2, respectiva-

\footnotetext{
a Acepran 0,2\% - UNIVET - São Paulo - SP.

- Tiopental sódico - Cristália - Itapira - SP.

- Propofol - Cristália - Itapira - SP.
}

mente, sendo portanto empregada dose de $6,25 \mathrm{mg} / \mathrm{kg}$ de tiopental sódico e $3,5 \mathrm{mg} / \mathrm{kg}$ de propofol, o qual foi diluído em solução salina a $0,7 \%$, para que o volume de ambos os anestésicos fosse exatamente o mesmo, de modo a ser utilizado $0,5 \mathrm{ml} / \mathrm{kg}$ de cada um dos agentes indutores. O tempo médio de aplicação dos referidos fármacos, para que a indução anestésica fosse estabelecida, foi de 25 segundos.

Parâmetros avaliados: freqüência cardíaca e ritmo cardíaco, por meio de eletrocardiograma, ${ }^{d}$ com uso de eletrodos adesivos $^{e}$ acoplados nos membros anteriores e inferiores, com leitura na segunda derivação de Eithoven; oximetria de pulso,' mediante a adaptação do sensor na língua dos animais; freqüência respiratória, pela inspeção dos movimentos torácicos; número de tentativas para a realização da IE; presença do reflexo de tosse; ocorrência de laringoespasmo; coloração das mucosas; necessidade de doses complementares para a intubação endotraqueal; grau de relaxamento da glote (escore de 0-2), onde 0=relaxamento ruim, sendo impossível a intubação endotraqueal sem a aplicação de doses adicionais do agente indutor; 1=relaxamento razoável, onde a intubação foi possível sem a administração de dose adicional do agente indutor, porém não foi realizada em primeira tentativa; 2 = bom, o relaxamento foi adequado, permitindo intubação endotraqueal em primeira tentativa.

Após a indução anestésica foi realizada a laringoscopia seguida de IE (Figuras 1 e 2), com uso de sonda endotraqueal descartável sem cuff. ${ }^{g}$ As sondas endotraqueais foram lubrificadas com lidocaína a $2 \%$ gel $^{\mathrm{g}}$ imediatamente antes da IE. O tamanho da sonda endotraqueal foi ajustado com o peso do animal sendo utilizado o número 3 para animais de até 2,5 kg e número 3,5 para animais acima desse peso corpóreo.

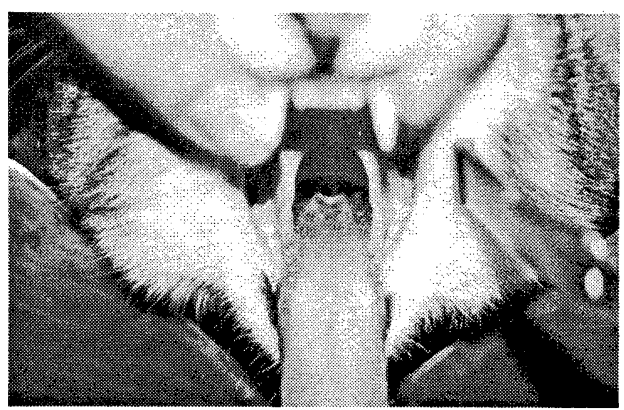

Figura 1: Cavidade orofaringea do gato

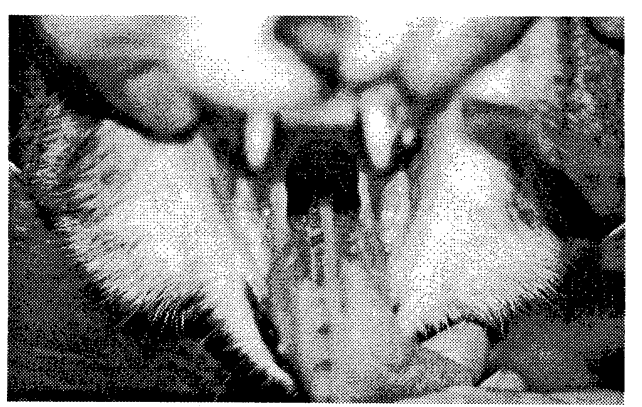

Figura 2: Intubação endotraqueal no gato

a Cardiotest EK 51 - PPG Biomedical Systems Division - Freiburg - Germany.

- Eletrodo para ECG AG/AGCL com gel

Oxímetro Cat. Mod.1001- JG Moriya - Ipiranga - SP.

${ }^{9}$ Rüsh

Xylestesin gel 0,5\% - Cristália - Itapira - SP. 


\section{Momentos avaliados}

M1 - Antes da indução anestésica: todos os parâmetros acima mencionados, com exceção da oximetria de pulso.

M2 - No momento da intubação endotraqueal: avaliação da FC e ritmo cardiaco.

M3 - 5 minutos após a intubação endotraqueal: oximetria de pulso e FR

Tabela 1 - Respostas dos pacientes à intubação endotraqueal

\begin{tabular}{cccc}
\hline & $\begin{array}{c}\text { G1 } \\
(n=10)\end{array}$ & $\begin{array}{c}\text { G2 } \\
(n=10)\end{array}$ & $\begin{array}{c}\text { G3 } \\
(n=10)\end{array}$ \\
\hline Reflexo de tosse & $10^{\mathrm{a}^{\mathrm{a}}}(100 \%)$ & $4^{\mathrm{b}}(40 \%)$ & $8^{\mathrm{b}}(80 \%)$ \\
\hline Laringoespasmo & $1^{\mathrm{a}}(10 \%)$ & $0^{\mathrm{a}}$ & $2^{\mathrm{a}}(20 \%)$ \\
\hline $\begin{array}{c}\text { Relaxamento } \\
\text { inadequado }\end{array}$ & $7^{\mathrm{a}}(70 \%)$ & $0^{\mathrm{b}}$ & $3^{\mathrm{ab}}(30 \%)$ \\
\hline $\begin{array}{c}\text { Intubação em 1 } \\
\text { tentativa }\end{array}$ & $3^{\mathrm{a}}(30 \%)$ & $8^{\mathrm{a}}(80 \%)$ & $5^{\mathrm{a}}(50 \%)$ \\
\hline
\end{tabular}

* Nas linhas: médias de grupos seguidas de letras minúsculas iguais nāo diferem entre si.

Para todos os animais a intubação endotraqueal foi realizada em decúbito dorsal, pelo mesmo anestesista, o qual fez a avaliação do grau de relaxamento de glote, segundo o escore proposto acima.

A análise estatística foi feita através de análise de variância de Friedman, teste $Q$ de Cochran's e análise de variância de Tukey. Os resultados foram considerados significativos para $p<0,05$.

\section{Resultados}

Os resultados obtidos demonstraram melhor qualidade de intubação endotraqueal com o uso do propofol isoladamente, em relação aos demais grupos (Tabela 1 ).

O reflexo de tosse foi menos evidente nos animais induzidos com o propofol (G2), em relação aos grupos induzidos com tiopental sódico (G1) ou tiopental sódico/propofol (G3) (40\%, $100 \%$ e $80 \%$ ), respectivamente.

Embora sem diferença significativa entre os grupos G2 e G3, o reflexo de tosse foi menos intenso para o grupo G2. No grupo $\mathrm{G} 1$, além de todos terem respondido com reflexo de tosse à inserção da sonda endotraqueal, em dois animais a tosse permaneceu por cinco minutos após a intubação endotraqueal, demonstrando-se muito exacerbada para os demais animais avaliados. Maior inibição do reflexo laringotraqueal foi observada nos animais do grupo G2, com mínima resposta dos animais ao estímulo produzido pela sonda endotraqueal. Para os animais do grupo G1 o reflexo laringotraqueal mostrou-se extremamente presente para todos os animais.

A intubação endotraqueal foi realizada com sucesso na primeira tentativa em $30 \%, 80 \%$ e $50 \%$, para os grupos $\mathrm{G1}$, G2 e G3, respectivamente.
O laringoespasmo foi observado em apenas um änimal do G1 e em dois animais do G3.

Dose adicional para realização da intubação endotraqueal foi necessária em apenas um animal do $\mathrm{G1}$, totalizando a dose de $21 \mathrm{mg} / \mathrm{kg}$.

Em nenhum dos grupos estudados foi observada ocorrência de taquicardia ou arritmias cardiacas subseqüentes à intubação endotraqueal.

Embora não tenha existido diferença significativa entre os grupos, a depressão respiratória foi mais evidente nos animais do G2, com episódios de apnéia transitória em dois animais.

\section{Discussão e conclusões}

Para que a intubação endotraqueal possa ser realizada é necessário que os reflexos da laringe sejam suprimidos pelo uso de anestésicos locais, bloqueadores neuromusculares ou plano anestésico profundo.

Alguns trabalhos realizados no homem têm demonstrado melhor qualidade na intubação endotraqueal e inserção da máscara laríngea com o uso do propofol, obtendo-se maior atenuação dos reflexos das vias aéreas (McKeating et al. 1988; Brown et al. 1991; Scanlon et al. 1993). No presente estudo, os resultados obtidos foram semelhantes aos observados por Scanlon et al. (1993), os quais compararam a qualidade de inserção da máscara laríngea no homem, com o uso do tiopental sódico em relação ao uso do propofol em doses equipotentes, com obtenção de menor incidência de tosse e laringoespasmo, melhor relaxamento de epiglote e maior depressão sobre o reflexo laringotraqueal com o uso do propofol. Embora no atual trabalho não tenha sido demonstrada diferença significativa em relação ao número de tentativas para realização da intubação endotraqueal, em apenas dois $(n=10)$ animais que foram tratados com propofol, foi necessária a segunda tentativa para a conclusão do procedimento, enquanto para os animais tratados com tiopental, somente, em três $(n=10)$ animais a intubação foi realizada com sucesso na primeira tentativa. Yeo et al. (2001) avaliaram o emprego da associação de tiopental sódico/propofol para a inserção da máscara laríngea no homem, com resultados semelhantes ao uso do propofol isoladamente, com a vantagem de redução de $45 \%$ do custo. No entanto, nesse trabalho, a associação de tiopental sódico/propofol não proporcionou melhor qualidade de intubação endotraqueal em relação ao uso do propofol isoladamente, porém essa associação demonstrou maior depressão sobre o reflexo laringotraqueal e menor incidência de tosse em relação ao uso do tiopental sódico isoladamente. Em estudo semelhante, realizado no cão, a indução anestésica com a associação de tiopental $(15 \mathrm{mg} / \mathrm{kg})$ ao propofol $(6 \mathrm{mg} / \mathrm{kg})$, em volume de $1: 1$, foi de qualidade similar, quando comparada ao uso isolado de cada um dos agentes indutores, nas referidas doses (Ko et al., 1999).

No homem, o estresse induzido pela laringoscopia no ato da intubação endotraqueal inclui taquicardia, hipertensão e arritmias (Malbty, 1994). No entanto, no presente estudo, os resultados inerentes à freqüência cardíaca e ritmo cardíaco foram similares aos observados por Cassu et al. (1999), os quais avaliaram a inserção da máscara laríngea ou intubação endotraqueal em gatos, com o uso do tiopental sódico, não 
sendo observadas taquicardia ou arrit nias subseqüentes à introdução da sonda endotraqueal, achado este contrário aos resultados observados em estudos realizados no homem, notando-se em alguns casos a ocorrência de arritmias cardíacas, hipertensão arterial e elevação da concentração plasmática de catecolaminas (Wood e Forrest, 1994; Fuji et al., 1995; Imai et al., 1995).

\section{Referências}

BROWN GW, PATEL N, ELLIS FR. Comparison of propofol and thiopentone for laryngeal mask insertion. Anaesthesia v. 46, p. 771-772, 1991.

CASSU RN, LUNA SPL, TEIXEIRA NETO FJ, BRAZ, JRC. O uso da máscara laríngea na anestesia geral inalatória em gatos sob respiração espontânea e controlada. Botucatu, 1999. 133 p. Dissertação (Mestrado em Anestesiologia Experimental) - Faculdade de Medicina - Unversidade Estadual Paulista.

CLARKE, K.W., HALL, L.W. A survey of anaesthesia in small animal practice. AVA / BSAVA report. Journal Association of Veterinary Anaesthesia. v. 17, p. 4-10, 1990.

FELDMAN, S., HARROP-GRIFFITHS, W., HIRSCH, N. Problems in anaesthesia: analysis and Management. Oxford: Heinemann, 1989.

FUIIIY., TANAKA, $H$., TOYOOKA, $H$. Circulatory responses to laryngeal mask airway insertion or tracheal intubation in normotensive and hypertensive patients. Canadian Journal of Anaesthesia. v. 42, p. 3236, 1995.

HEAVNER, J. E. Cardiac dysrhythmias induced by infusion of local anesthetics into the lateral cerebral ventricle of cats. Anaesthesia and Analgesia. v. 65, p. 133, 1986.

IMAI, M., MATSUMURA, C., HANAOKA, Y., KEMMOTSU, O. Comparison of cardiovascular responses to airway management: fiberoptic intubation using a new adapter, laryngeal mask insertion, or convencional laryngoscopic intubation. J. Clin. Anaesth. v. 7, n. 1, p. 14-18, 1995.
Perante as condições experimentais realizadas neste estudo, conclui-se que o propofol permitiu maior facilidade para a intubação endotraqueal em gatos em relação ao tiopental sódico e à associação tiopental sódico/propofol, sugerindo que o propofol pode ser usado como agente anestésico indutor para gatos que serão submetidos à intubação endotraqueal, minimizando possíveis efeitos indesejáveis induzidos pela mesma.

MALBTY, J.R. The laryngeal mask airway in anaesthesia. Canadian Journal of Anaesthesia, v. 41, p. 888-893, 1994.

MCKEATING K, BALI IM, DUNDEE JW. The effects of thiopentone and propofol on upper airway integrity. Anaesthesia, v. 43, p. 638-40, 1988.

REX, M.A.E. Anatomy. In: HALL, L.W. and TAYLOR, P.M. Anaesthesia of the cat. Baillière Tindall, 1994, Cap 1, p.1-11.

SCANLON P, CAREY M, POWER M. Patiente response to lanyngeal mask insertion after induction of anaesthesia with propofol or thiopentone. Can J Anaesth. v. 40, p. 816-818, 1993.

SOMERVILLE, M., KARLSSON, J.A., RICHARDSON, P.S. The effects of local anaesthetic agents upon mucus secretion in the feline trachea in vivo. Pulm Pharmacologic. v. 3, p. 93-101, 1990.

TAYLOR, P.M. Use of Xylocaine pump spray for intubation in cats. Veterinary Record. v. 130, p. 583, 1992. Veterinary use of Xylocaine spray. British Journal of Anaesthesia. v. 70, p. 113, 1993.

. Accidents and Emergencies. In: HALL, L.W. and TAYLOR, P.M. Anaesthesia of the cat. Baillière Tindal, 1994, Cap 12, p.249-272. WATSON, A.K. Use of Xylocaine pump spray for intubation in cats. Vet Rec. v. 130, p. 455, 1992.

WOOD, M.L.B., FORREST, E.T.S. The haemodynamic response to the insertion of the laryngeal mask airway: a comparison with laryngoscopy and tracheal intubation. Acta Anaesthesiologica Scandinava. v. 38, p. 510-513, 1994.

YEOKS, KUA SW, TEOH GS, ONSIONG MK. The use of thiopentone admixture for laryngeal mask airway insertion. Anaesth Intensive Care, v. 29, p. $38-42,2001$. 